\title{
Properties of kinematic singularities
}

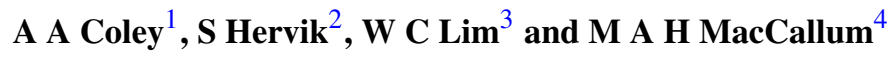 \\ ${ }^{1}$ Department of Mathematics \& Statistics, Dalhousie University, Halifax, Nova Scotia B3H 3J5, \\ Canada \\ ${ }^{2}$ Department of Mathematics and Natural Sciences, University of Stavanger, N-4036 Stavanger, \\ Norway \\ 3 Albert-Einstein-Institut, Am Mühlenberg 1, D-14476 Potsdam, Germany \\ ${ }^{4}$ School of Mathematical Sciences, Queen Mary University of London, E1 4NS, UK \\ E-mail: aac@mathstat.dal.ca, sigbjorn.hervik@uis.no,wclim@aei.mpg.de and \\ m.a.h.maccallum@qmul.ac.uk
}

Received 8 July 2009, in final form 4 September 2009

Published 6 October 2009

Online at stacks.iop.org/CQG/26/215008

\begin{abstract}
The locally rotationally symmetric tilted perfect fluid Bianchi type V cosmological model provides examples of future geodesically complete spacetimes that admit a 'kinematic singularity' at which the fluid congruence is inextendible but all frame components of the Weyl and Ricci tensors remain bounded. We show that for any positive integer $n$ there are examples of Bianchi type $\mathrm{V}$ spacetimes admitting a kinematic singularity such that the covariant derivatives of the Weyl and Ricci tensors up to the $n$th order also stay bounded. We briefly discuss singularities in classical spacetimes.
\end{abstract}

PACS numbers: $98.80 . J k, 04.20 .-\mathrm{q}$

\section{Introduction}

A cosmological model $(\mathcal{M}, \mathbf{g}, \mathbf{u})$ is defined by specifying the spacetime geometry, determined by a Lorentzian metric $\mathbf{g}$ defined on the manifold $\mathcal{M}$, a family of fundamental observers, whose congruence of worldlines is represented by the 4-velocity field $\mathbf{u}$, and some appropriate matter content. The covariant derivative $u_{a ; b}$ of the 4-velocity field can be decomposed into kinematic variables according to

$$
u_{a ; b}=\sigma_{a b}+\omega_{a b}+H\left(g_{a b}+u_{a} u_{b}\right)-\dot{u}_{a} u_{b},
$$

where $\sigma_{a b}$ is the rate of shear tensor, $\omega_{a b}$ is the vorticity tensor, $H$ is the Hubble scalar and $\dot{u}_{a}$ is the acceleration vector.

In a recent paper [1], the future ${ }^{5}$ asymptotic dynamics as experienced by the perfect fluid observer in spatially homogeneous ( $\mathrm{SH})$ Bianchi cosmologies with a tilted perfect fluid with

5 'Future' means 'in the direction of expansion'. 
linear equation of state $p=(\gamma-1) \mu$ was examined. It was found that given a Bianchi type and a large enough value of the equation-of-state parameter, $\gamma$, the perfect fluid observer can encounter a singularity in the sense that the fluid congruence becomes inextendible after a finite proper time. This is not a true spacetime singularity, since the causal geodesics are complete. Indeed, observers moving orthogonally to the SH hypersurfaces, and therefore geodesically, would not encounter a spacetime singularity in the future [2]. The fluid does not follow geodesics: it is accelerated. The pathology of the flow congruence is therefore called a kinematic singularity ${ }^{6}$ [3]: it is accompanied by an extreme tilt limit (i.e. the fluid observers' motion becomes asymptotically null with respect to the $\mathrm{SH}$ observers). It has the properties that (i) the proper time needed to reach the kinematic singularity is finite, (ii) the Hubble scalar $H$ and some other kinematic quantities diverge, and (iii) the matter density tends to zero (i.e. the kinematic singularity is not a matter singularity). Since the source is a perfect fluid with a linear equation of state, this last means that all components of the Ricci tensor in the comoving frame tend to zero.

The Weyl parameter, $\mathcal{W}$, is defined by [4]

$$
\mathcal{W}=\frac{W}{H^{2}}, \quad \text { where } \quad W^{2}=\frac{1}{6}\left(E_{a b} E^{a b}+H_{a b} H^{a b}\right) .
$$

The phenomenon of Weyl curvature dominance occurs when $\mathcal{W} \rightarrow \infty$, which can happen in Bianchi type VII $_{0}$ and VIII cosmologies $[4,5]$. The Weyl parameter can be defined with respect to different invariantly defined systems of observers and, particularly in Bianchi models with a tilted perfect fluid, with respect to the fluid observers and the spatially homogeneous observers. Although the limits of the Weyl parameter along these two congruences of worldlines are in principle different, it has been conjectured that in general they both tend to zero, constant or infinity together [3]. However, note that this does not occur in the exceptional locally rotationally symmetric (LRS) Bianchi type V models, due to cancellation of terms.

Suppose that the worldlines of a congruence are incomplete and inextendible. For brevity, we shall talk about limiting behaviour on the curves as if it were possible to attach endpoints to them, although there are difficulties in doing so in such a way as to construct a sensible boundary to the spacetime [6]. If a curvature scalar blows up as the endpoint is approached, the endpoint may be a true spacetime singularity, as discussed in section 3 (the alternative is that the blow up occurs at infinity along causal geodesics). If the matter (or Ricci) tensor blows up, we call this a matter singularity, while if only the Weyl tensor is singular we call it a Weyl singularity ${ }^{7}$ (we could use the term 'conformal singularity', following [7], but we wish to avoid confusion with the 'conformal singularity' in the context of isotropic singularities [8]).

If at least one component of the Weyl curvature tensor (with respect to the orthonormal frame of an observer travelling along the congruence) diverges, we call this Weyl blow-up ${ }^{8}$. Another possibility is that the matter tensor and Weyl tensor both converge as the endpoint is approached, but some kinematic quantities (most importantly $H$ ) diverge, i.e. a

6 So called at some risk of confusion with true spacetime singularities as defined in section 3: the word 'singularity' is retained here because the two concepts have in common the idea of incomplete inextendible causal curves, while differing in whether these must be geodesic or can accelerate.

7 This definition of a Weyl singularity is a more natural terminology for the case of a true spacetime singularity-it differs from the definition of a 'Weyl singularity' utilized in [3], which we shall refer to here as Weyl blow-up in finite proper time.

8 Note that it is possible that all Weyl scalar invariants converge, and the Weyl tensor is non-singular, but that Weyl blow up occurs, which implies that one observer may experience Weyl blow-up while another does not. This can, and in general will, happen when the Weyl tensor invariants have a non-zero limit along an observer's worldline and in the limit that observer's motion becomes lightlike with respect to the frame fixed by the Weyl tensor (e.g., see [9, chapter 4]). 
kinematic singularity occurs. Thus, relative to a timelike congruence, a kinematic singularity is characterized by the blow-up of one or more kinematic variables in finite proper time, while all components of the Weyl and Ricci tensors remain bounded.

On approaching to an extreme-tilt limit, the fluid observers in the tilted Bianchi cosmologies may or may not experience Weyl blow-up, and so the limit may or may not be a kinematic singularity [3]. The LRS Bianchi V models provide examples of non-spacetime singular models both with Weyl blow-up and with a kinematic singularity. When studying the properties of a true singularity attention is usually focussed on the behaviour of the Weyl tensor and the Ricci tensor. However, there are examples in which Weyl blow-up does not indicate a singularity and kinematic quantities are useful in indicating a kinematic singularity. An important related question, which we shall address here, is whether a kinematic singularity is always associated with the divergence of the components of the covariant derivatives of the Riemann tensor.

\section{LRS bianchi type V model}

The LRS Bianchi type V model provides examples of non-spacetime singular models which are geodesically complete and show Weyl blow-up and kinematic singularities (and are such that all scalar invariants involving the Weyl and Ricci tensors, including mixed invariants, are bounded).

First, we give a brief sketch of the derivation of the LRS Bianchi V limits near the future asymptotic state $M^{-}$. We use the dimensionless time variable $\tau$, defined by equation (2.10) of [10]:

$$
\frac{\mathrm{d} t}{\mathrm{~d} \tau}=\frac{1}{H}
$$

where $t$ is the proper time along the spatially homogeneous congruence. Using the evolution and constraint equations (2.11) and (2.12) of [10], we determine the linearized equations at $M^{-}$for the following variables:

$$
H, \Sigma_{+}, \Omega, 1-v^{2}, \quad 1-A^{2} .
$$

We then compute the decay rates for $E_{+}$and $\mathcal{W}$ through their algebraic expressions and use the boost formulae to obtain the decay rates for $\hat{H}$ and $\hat{E}_{+}$. Finally, we obtain the decay rates for $\hat{W}$ and $\hat{\mathcal{W}}$.

We take this opportunity to correct some computational errors made in [3]. Equations (21), (23), (27) and (28) should read, respectively,

$$
\begin{aligned}
& H \approx H_{0} \mathrm{e}^{-\tau}, \quad W \approx 2 H_{0}^{2}\left|\Sigma_{+0}\right| e^{-6 \tau}, \quad \mathcal{W} \approx 2\left|\Sigma_{+0}\right| e^{-4 \tau} \\
& E_{+}=\left(H+\sigma_{+}\right) \sigma_{+}+\frac{1}{6} \frac{\gamma \mu}{G_{+}} V^{2} \\
& \hat{W}=W \approx 2 H_{0}^{2}\left|\Sigma_{+0}\right| e^{-6 \tau} \\
& \hat{\mathcal{W}} \approx \frac{9}{8}(2-\gamma)^{2} \Gamma_{0}^{-2}\left|\Sigma_{+0}\right| \exp \left(\frac{-2(3 \gamma-2)}{2-\gamma} \tau\right) .
\end{aligned}
$$

The computational errors do not affect the conclusions of [3].

In [3] it was shown that in the exceptional LRS Bianchi V models $H \rightarrow 0, W \rightarrow 0$, $\mathcal{W} \rightarrow 0$ as $\tau \rightarrow \infty$ for $6 / 5<\gamma<2$. However, in the fluid frame $\hat{H} \rightarrow \infty$ as $\tau \rightarrow \infty$ for $4 / 3<\gamma<2$, but $\hat{W} \rightarrow 0, \hat{\mathcal{W}} \rightarrow 0$ for $6 / 5<\gamma<2$ and hence do not blow up. Therefore, in this model there are kinematic singularities.

In [7] it was claimed that the future singularity in the LRS Bianchi type V model is a 'conformal singularity' (figure 7, right-hand side of 7(i) and (ii), the bottom spacetime diagram 
and table). As mentioned in [3], this is not the case, since the Weyl components tend to zero. What happens instead is that some kinematic variables blow up in finite time ${ }^{9}$. Singularities were classified in [12] as 'curvature singularities', 'intermediate singularities' and 'locally extendible singularities'. Weyl blow-ups where at least one of the Weyl scalars also blows up correspond to curvature singularities, Weyl blow-ups with bounded Weyl scalars (and Ricci scalars) correspond to intermediate singularities, and kinematic singularities correspond to locally extendible singularities, or points at infinity. In our examples, (i) the fluid worldlines are certainly not extendible, due to the blow up of the kinematic variables, and are thus not 'locally extendible' in the sense of Clarke [13], (ii) we wish to use terminology that better describes the type of behaviour under discussion.

The kinematic quantities are given by ratios of Cartan invariants, using a co-moving frame as the canonical one [14]: more specifically, they are given by dividing the derivatives of the tracefree Ricci tensor by $(\mu+p)$, a multiple of its one independent non-zero component. Hence, assuming we do not have $(\mu+p) \equiv 0$, kinematic singularities arise because (i) both $(\mu+p)$ and the first derivatives tend to zero, but the ratio tends to infinity, or (ii) $(\mu+p) \rightarrow 0$ while some first derivative of the matter tensor remains finite and non-zero, or (iii) the first derivative blows up, possibly while $(\mu+p)$ remains finite. A kinematic singularity might also be accompanied by other divergences; e.g. a divergence of a component of the first covariant derivative of the Weyl tensor. In order to investigate this here, we only need to consider the LRS Bianchi type $\mathrm{V}$ model, since more than one possible behaviour occurs in this particular example.

\subsection{Weyl derivatives}

For higher derivatives of the Weyl scalar, we first note that $H$ has the slowest decay rate $e^{-\tau}$ among the kinematic variables. Next, we note that a partial derivative with respect to proper time, denoted by an overdot, is $H$ times the partial derivative with respect to $\tau$. Since the decay rates are exponential in $\tau$, the time derivative of a variable has the same decay rate as $H$ times the variable to be differentiated.

Dimensional consistency implies that the algebraic terms in a covariant derivative must be a single power of one of the kinematic variables times the scalar variable (or tensor components) to be differentiated. Since $H$ is the slowest decaying kinematic variable, the slowest decay rate for the algebraic term is the same as the decay rate for the time derivative above. We can then apply the same reasoning for higher derivatives.

To convert to the fluid frame components, an orthonormal frame component (not coordinate component) of a rank- $n$ tensor obtained by contraction with a unit vector in the fluid flow plane will be multiplied with $\Gamma^{n}$ due to the boost formula for the frame vectors.

The LRS Bianchi type $\mathrm{V}$ model has only one non-zero Weyl component, $E_{+}$, so consequently $E_{+}$is a scalar invariant and its covariant derivatives are simply derivatives of $E_{+} . E_{+}$has the decay rate $e^{-6 \tau}$. In this model, all of the scalar curvature invariants are bounded: $C^{2} \equiv C_{a b c d} C^{a b c d} \sim E_{+}^{2}$ and $C_{, 1}{ }^{2} \equiv C_{a b c d ; e} C^{a b c d ; e} \sim \dot{E}++^{2} \sim\left(H E_{+}\right)^{2} \rightarrow 0$, and $C_{, n}{ }^{2} \equiv C_{a b c d ; e_{1} \ldots e_{n}} C^{a b c d ; e_{1} \ldots e_{n}} \sim\left(H^{n} E_{+}\right)^{2} \sim \exp [-2(n+6) \tau] \rightarrow 0$ (the higher derivatives converge even faster).

Let us consider individual components of the Weyl tensor and its derivatives in the fluid frame $^{10}$. The boost factor $\Gamma$ has the growth rate $\exp \left[\frac{(5 \gamma-6)}{2-\gamma} \tau\right]$, and the Hubble scalar $\hat{H}$ of the fluid frame has the growth rate $\hat{H} \sim \Gamma H \sim \exp \left[\frac{2(3 \gamma-4)}{2-\gamma} \tau\right]$. These growth rates will be key to

9 The stability of the properties of the Bianchi V models (within the class of all spatially homogeneous models) has been studied by Siklos [11].

${ }^{10}$ Note that Hubble-normalized quantities such as for example, $\hat{E}_{+} / \hat{H}^{2}$, all tend to zero. 
the blow up of the derivatives. The first-order derivatives of $E_{+}$in the fluid frame have leading order of $\dot{\hat{C}} \sim \Gamma H E_{+} \sim \hat{H} E_{+} \sim \exp \left[\frac{4(3 \gamma-5)}{2-\gamma} \tau\right]$. They blow up if $\gamma>5 / 3$. The $n$ th-order derivatives (both covariant and partial) of $E_{+}$have leading order of $\hat{H}^{n} E_{+}$. They blow up if

$$
\gamma>\frac{2(2 n+3)}{3(n+1)} \text {. }
$$

A sample of $\gamma$ thresholds for each $n$ is given by

\begin{tabular}{|c|ccccc|}
\hline$n$ & 1 & 2 & 3 & 4 & 5 \\
\hline$\gamma$ & $5 / 3$ & $14 / 9$ & $3 / 2$ & $22 / 15$ & $13 / 9$ \\
\hline
\end{tabular}

Another way to say this is that for a fixed $\gamma$, the derivatives blow up for

$$
n>\frac{3(2-\gamma)}{3 \gamma-4}
$$

In the Bianchi $\mathrm{V}$ example, for any $n$ there exists a range of values for $\gamma$ such that the Weyl tensor and its first $n$ covariant derivatives all stay bounded, and other values for which the Weyl tensor and/or its derivatives at some orders less than $n$ blow up. Therefore, at a kinematic singularity both behaviours are possible, and the notion of blow up of kinematical quantities is useful.

\subsection{Ricci derivatives}

Similarly, for the Ricci scalar, or equivalently, $\hat{\mu}$, which has the decay rate $\hat{\mu} \sim \Gamma^{-2} \mu \sim$ $\exp \left[\left(-2 \frac{(5 \gamma-6)}{2-\gamma}-6\right) \tau\right]$, the $n$ th-order derivatives (both covariant and partial) of $\hat{\mu}$ have leading order of $\hat{H}^{n} \hat{\mu}$. They blow up if

$$
\gamma>\frac{4 n}{3 n-2}
$$

for $n=3,4, \ldots$. We omit $n=1$ and $n=2$ because the $\gamma$ thresholds are $\geqslant 2$. A sample of $\gamma$ thresholds for each $n$ is given by

\begin{tabular}{|c|ccc|}
\hline$n$ & 3 & 4 & 5 \\
\hline$\gamma$ & $12 / 7$ & $8 / 5$ & $20 / 13$ \\
\hline
\end{tabular}

Equivalently, the derivatives blow up for

$$
n>\frac{2 \gamma}{3 \gamma-4} \text {. }
$$

As an example, consider $\gamma=5 / 3$. In this case, the components of both the Weyl tensor and the Ricci tensor tend to zero. The components of the first derivative of the Weyl tensor are bounded, but the components of the second derivative of the Weyl tensor are unbounded, while the derivatives of the Ricci tensor up to third order tend to zero and the components of the fourth derivative of the Ricci tensor blow up.

\section{Discussion}

Due to these results, and given the recent tendency to use imprecise terminology, particularly in the physics literature, perhaps it would be useful to briefly review singularities in classical general relativity.

The existence of a spacetime singularity is defined by the inextendibility of causal (that is, null or timelike) geodesics [15, 16]. More precisely, a manifold is said to have a spacetime 
singularity if its maximal development is geodesically incomplete. The problem with defining a boundary point of spacetime at which the singularity can be said to occur is that the geometry is singular there, so that the singularity cannot be a regular spacetime point. So these points are never in the manifold initially considered, but the definition requires its maximal development. This implies that the identification of a real singularity depends on the criteria chosen for extendibility; for example, if discontinuous extensions were allowed, then the Friedmann solutions would be extendible and so would not have real singularities [6].

For the existence of solutions of the geodesic equation, the metric must be $C^{1}$ and for these solutions to depend continuously on initial position and direction, it must be $C^{2-}$ (see [17] $)^{11}$. There are various other differentiability conditions (on the metric or on the Riemann tensor components) that could be used (together with other additional conditions). Perhaps the most widely used inextendibility assumptions are that the extended metric be $C^{N}$, where $N$ is the minimum differentiability needed for the existence of a unique maximal development of the Einstein equations from a set of initial data [6]. Using this definition the class of non-singular spacetimes includes at least some shock waves where there is a discontinuity in the Riemann tensor, but not impulse waves. Indeed, any mathematical model of the inhomogeneous matter model is likely to allow shock waves to form (and non-analyticity will cause high-enough derivatives of Riemann tensor to blow up).

Once a spacetime singular point has been constructed, its properties are of interest. There has been much work on the occurrence and possible natures of singularities thus defined [6]. Singularities can be classified according to the boundedness or divergence of scalar polynomials of the Riemann tensor (and its covariant derivatives). A scalar polynomial curvature singularity is the end point of at least one curve on which a scalar polynomial in the metric and the Riemann tensor takes unboundedly large values (and hence some Riemann tensor component is unbounded in any tetrad, and not just in parallelly propagated ones). Archetypical examples are the Schwarzschild solution with a timelike curvature singularity in which the Kretschmann scalar (which is equivalent to the square of the Weyl curvature in this case) diverges, and the Friedmann cosmological spacelike initial singularity in which the Ricci scalar diverges.

Non-scalar polynomial curvature singularities (or 'intermediate singularities') are singularities where all the scalar polynomials in the Riemann tensor are bounded, and on every curve ending at the singularity (nonparallel) frames can be chosen in which the components of the Riemann tensor are bounded. Examples include the cosmological 'whimpers' found in anisotropic SH tilting fluid cosmologies [12]. In addition, a pp-wave spacetime can be singular, but all of its scalar invariants are identically zero [9]. These types of singularities are more complicated since they need not be encountered by all local observers.

It should be noted that the presence of a diverging scalar invariant is not sufficient to prove the presence of a spacetime singularity (and it may not be necessary). In the Kruskal-Szekeres spacetime, which is the maximal analytic extension of the Schwarzschild metric, the scalar polynomial formed from the first derivatives of the curvature, $R^{a b c d ; e} R_{a b c d ; e}$, vanishes exactly at the horizon [18]. This can be used to illustrate that the existence of invariants that blow up does not necessarily imply a spacetime singularity (for example, consider $1 / R^{a b c d ; e} R_{a b c d ; e}$ ) [19].

If a spacetime is geodesically complete, it may still be pathological or singular in some sense, e.g., as in the examples above, such a spacetime may still admit non-geodesic (i.e., accelerating) timelike curves that are incomplete. For this reason no definition distinguishing singular and non-singular spacetimes by geodesic completeness alone can

${ }^{11}$ In this and subsequent statements, we use function spaces defined by differentiability and Lipshitz conditions. Somewhat weaker conditions using Sobolev spaces actually suffice; e.g., see [6]. 
be totally satisfactory [15]: in particular, worldlines that represent observers ought to be complete, and geodesic completeness does not prevent other curves from 'escaping' the spacetime. Classic examples include Geroch's rocket with bounded acceleration, Misner's example of trapped curves in a torus, and tilting perfect fluid observers (in the future direction) in LRS Bianchi V models with unbounded acceleration. There are also examples in Minkowski spacetime in which accelerating curves can be found so that the expansion blows up in a finite proper time, and hence no meaningful extension can be defined [6]. It is of use to define singular spacetimes in such a way that the pathological behaviour is clear and meaningful (and carries information on how the singular behaviour occurs) [19]. Spacetimes such that all curves ending at a singularity have the property that the components of the Riemann tensor in a parallelly propagated frame remain bounded along the curve, have been called 'quasiregular' [6].

Therefore, a pathological spacetime that does not admit a spacetime singularity must have a structure defined on the spacetime manifold that is singular, and this may or may not be accompanied by a divergence of the components of the curvature tensor or its derivatives. There could be (i) pathologies of mathematical structures defined on the spacetime manifold (e.g., poorly behaved congruences or spinor structure, conical singularities), or (ii) pathologies of physical structures defined on the spacetime manifold (e.g., worldlines of matter). Singularities for which physical quantities diverge are perhaps of more importance. However, in the context of current theories of high-energy physics, lines may be blurred between geometrical and physical singularities; e.g., in supergravity theory additional fields such as the dilaton and form fields are gravitational degrees of freedom.

In the previous section, we have shown that Bianchi $\mathrm{V}$ models provide examples of such pathologies which are not spacetime singularities, where components of high enough order covariant derivatives of the Riemann tensor blow up. In this case, the 'singularities' in the derivatives are in some sense at infinity, although reachable in finite time by the fluid. The example discussed by Musgrave and Lake [20] shows that one can have similar blowup of derivatives, without a spacetime singularity, at the centre of a spherically symmetric configuration.

We now answer the question raised at the end of the introduction. For the fluid congruence in the LRS Bianchi type V models, the kinematic singularity, which occurs at the asymptotic state $M^{-}$for $\frac{4}{3}<\gamma<2$, is associated with the blow-up of components of covariant derivatives of the Weyl tensor of order $n>\frac{3(2-\gamma)}{3 \gamma-4}$, and of the Ricci tensor of order $n>\frac{2 \gamma}{3 \gamma-4}$. That is, at the kinematic singularity, while the components of the Riemann tensor and its low-order covariant derivatives are bounded, those of its high-order covariant derivatives blow up. We speculate that this is also true in general.

In the LRS Bianchi type V models, the quantities which blow up are Cartan invariants defined by taking a frame defined by the fluid motion (up to the spacetime isotropy). In [21] it was shown that for spacetimes not in the Kundt class the components of the curvature tensors are, at least in principle, determined by the polynomial curvature invariants. The components correspond to roots of a set of characteristic polynomials; consequently, assuming that the algebraic type does not change, the components will blow up if and only if the polynomial curvature invariants blow up. Therefore, except in Kundt spacetimes, there exists a Cartan invariant that blows up if and only if there exists a polynomial curvature invariant that blows up. Hence our results show that, in general, one or more scalar polynomial invariants will blow up ${ }^{12}$.

12 To prove this rigorously it is necessary to assume that not only the algebraic types (of the curvature tensor and its covariant derivatives) change in the appropriate limit, but also that the differences between particular eigenvalues of certain projective operators do not approach zero in this limit. 
Finally, there is the issue of the possible resolution of classical singularities within quantum theory. At Planck scale curvatures, the character of gravity may change radically due to its underlying quantum nature. It is expected that singularities will be 'smoothed out' or 'resolved' in the correct theory of quantum gravity. For example, it is possible that there are regular solutions of the Dirac equation that can be extended through any classical singularity, and since strings experience the spacetime only through the sigma model, spacetimes which are singular in general relativity can be completely nonsingular in string theory [22].

\section{Acknowledgment}

This work was supported, in part, by NSERC of Canada.

\section{References}

[1] Coley A A, Hervik S and Lim W C 2006 Class. Quantum Grav. 233573 Coley A A, Hervik S and Lim W C 2006 Phys. Lett. B 638310 Coley A A and Hervik S 2005 Class. Quantum Grav. 22579

[2] Rendall A D 1995 Math. Proc. Camb. Phil. Soc. 118511

[3] Lim W C, Coley A A and Hervik S 2007 Class. Quantum Grav. 24595

[4] Wainwright J, Hancock M J and Uggla C 1999 Class. Quantum Grav. 162577

[5] Hervik S, van den Hoogen R J, Lim W C and Coley A A 2006 Class. Quantum Grav. 23845 Hervik S and Lim W C 2006 Class. Quantum Grav. 233017

[6] Tipler F J, Clarke C J S and Ellis G F R 1980 General Relativity and Gravitation: One Hundred Years After the Birth of Albert Einstein vol 2 ed A Held (New York: Plenum)

[7] Collins C B and Ellis G F R 1979 Phys. Rep. 5665

[8] Anguige K and Tod K P 1999 Ann. Phys., NY 276257 Goode S W, Coley A A and Wainwright J 1992 Class. Quantum Grav. 9445

[9] Stephani H, Kramer D, MacCallum M A H, Hoenselaers C A and Herlt E 2003 Exact Solutions of Einstein's Field Equations 2nd edn (Cambridge: Cambridge University Press)

[10] Hewitt C and Wainwright J A 1992 Phys. Rev. D 464242

[11] Siklos S T C 1978 Commun. Math. Phys. 58255

[12] Ellis G F R and King A R 1974 Commun. Math. Phys. 38119

[13] Clarke C J S 1973 Commun. Math. Phys. 32205

[14] Pareja M J and MacCallum M A H 2006 Class. Quantum Grav. 235039

[15] Geroch R 1968 Ann. Phys., NY 48526

[16] Wald R M 1984 General Relativity (Chicago: University of Chicago Press)

[17] Hawking S W and Ellis G F R 1973 The Large-Scale Structure of Spacetime (Cambridge: Cambridge University Press)

[18] Karlhede A, Lindström U and Åman J E 1982 Gen. Rel. Grav. 14569

[19] MacCallum M A H 2006 Gen. Rel. Grav. 381887

[20] Musgrave P and Lake K 1995 Class. Quantum Grav. 12 L39

[21] Coley A A, Hervik S and Pelavas N 2009 Class. Quantum Grav. 26025013 (arXiv:0901.0791)

[22] Horowitz G T 2005 New J. Phys. 7201 (arXiv:gr-qc/0410049) 